\title{
Downregulating forkhead box M1 inhibits proliferation by inhibiting autophagy in the sw480 cell line
}

\author{
SHIBIAO ZHONG ${ }^{1 *}$, AIYAN ZHOU ${ }^{2 *}$, FANGHUA QI $^{2}, \mathrm{ZHEN} \mathrm{LI}^{1}$, \\ ZEYAN YU ${ }^{1}$, YONGCHAO LU ${ }^{2}$ and XUDONG $\mathrm{LIU}^{3}$ \\ ${ }^{1}$ Department of Anorectal Surgery, Minzu Hospital of Guangxi Zhuang Autonomous Region, Nanning, \\ Guangxi Zhuang Autonomous Region 530001; ${ }^{2}$ Department of Traditional Chinese Medicine, \\ Shandong Provincial Hospital Affiliated to Shandong University, Jinan, Shandong 510080; ${ }^{3}$ Department \\ of Pain, Qilu Hospital of Shandong University, Jinan, Shandong 250000, P.R. China
}

Received March 1, 2017; Accepted March 30, 2017

DOI: $10.3892 /$ br.2017.915

\begin{abstract}
Forkhead Box M1 (FoxM1) is one of the most important oncogenes, and overexpression of FoxM1 has been reported in many cancers, including colon cancer. In the present study, the authors attempted to reveal the mechanism underlying its effects on proliferation through autophagy in the sw480 cell line. FoxM1 is knocked down through short hairpin (sh)RNA in the sw480 cell line. A series of experiments were conducted to examine it function on proliferation and LC3 and P62 were used to measure level of autophagy. Autophagy in the shFoxM1 cell was demonstrated as significantly inhibited compared with the negative control. Additional auto-fluex was also tested, downregulation of FoxM1 served the same role as BA1 in autophagy. Furthermore, downregulating FoxM1 inhibited cell proliferation in the sw480 cell line.
\end{abstract}

\section{Introduction}

Colon cancer is the third most frequently diagnosed cancer and one of the leading causes of cancer-related deaths worldwide $(1,2)$. Many studies have attempted to demonstrate its effects on tumorigenesis, however, further investigation is still required.

Forkhead Box M1 (FoxM1), a member of the Fox protein family characterized by a conserved winged-helix DNA binding domain, promotes cell cycle progression by inducing both transition from $\mathrm{G} 1$ to $\mathrm{S}$ phase, and transition from $\mathrm{G} 2$ to M phase (3). Previous studies demonstrated that overexpression

Correspondence to: Dr Yongchao Lu, Department of Traditional Chinese Medicine, Shandong Provincial Hospital Affiliated to Shandong University, 324 Jingwu Road, Jinan, Shandong 510080 , P.R. China

E-mail: luyongchaopaper@163.com

*Contributed equally

Key words: shFoxM1, autophagy, proliferation, colon cancer, sw480 cell line of FoxM1 is observed in a wide variety of cancers, including breast, ovarian, colon, liver, pancreatic, cervical and gastric cancers (4-6). Moreover, expression of FoxM1 is correlated with a clinically aggressive, drug-resistant, cancer phenotype and poor patient survival in many cancers (7). FoxM1 is regulated by oncogenic signals, including many crucial factors, such as the p53 tumor suppressor protein, which is mutated in half of human cancers (8). FoxM1 is considered to be one of the most important targets within the oncogenic pathway. Many studies of FOXM1 in the tumorigenesis of colon cancer have been conducted, more detailed research is needed, as its relation to autophagy is still unclear.

Autophagy was first reported by Christian de Duve at the International Lysosomal Conference in 1963. It refers to how some protein and organelles were packed into lysosome to degrade, and that the degradation products may be recycled to produce energy. It is widely accepted that autophagy is a means of self-protection when the cell is near death $(9,10)$. The autophagy process is a series of gradual evolution of autophagy structure. Once the autophagy is induced, the isolation membrane and phagophore are formed to combine with the protein and organelle. The isolation membrane extends and wraps the enclosed cytoplasmic component to form a bilayer membrane. Structure-autophosome, autophosome combines with lysosome to form autopholysome where the enclosed cytoplasmid is degraded $(9,10)$.

Autophagy is induced by a series of autophagy-related proteins (Atg). LC3 is a marker protein on the autophagosome. There are two forms of LC3 protein in the cell: LC3-I and LC3-II. LC3 is cleaved into LC-3I by Atg4 protease, LC3-I scattered in the plasmid, LC3-I and phosphatidylethanolamine, phycoerythrin is coupled to form LC3-II when the autophagosome is formed. Unlike other Atgs, LC3-II is located on the membrane of the autophagy structure steadily, so it is used as marker to reflect the level of autophagosomes $(11,12)$.

\section{Materials and methods}

Reverse transcription-quantitative polymerase chain reaction. A total of five frozen tumors and paired normal tissues stored at $-80^{\circ} \mathrm{C}$ are enrolled to test the expression level of 
FoxM1 mRNA, $\beta$-actin was used as a control. Total RNA was extracted with TRIzol reagent, reverse transcription was performed using oligo dT primers and SuperScript III RT (Invitrogen; Thermo Fisher Scientific, Inc., Waltham, MA, USA).

Cell lines, culture conditions. The human sw480 cell line was obtained from the American Type Culture Collection (Manassas, VA, USA). All cell lines were cultured in RPMI-1640 Medium supplemented with 10\% fetal bovine serum. Cells were cultured at $37^{\circ} \mathrm{C}$ in a humidified incubator with $5 \% \mathrm{CO}_{2}$.

Lentivirus production. Short hairpin (sh)RNA and a negative control were transfected into HEK293T by Lipofectamine ${ }^{\circledast} 3000$ to produce the lentivirus following manufacturer's protocol. The shRNA and negative control were transfected into HEK293T cells when the cell density was $95-99 \%$. The medium was collected following incubating for $72 \mathrm{~h}$ at $37^{\circ} \mathrm{C}$ in a humidified incubator with $5 \% \mathrm{CO}_{2}$. sw480 cells were seeded into a six-well plate, and, following incubating for $12 \mathrm{~h}, 1 \mathrm{ml}$ lentivirus medium with $1 \mathrm{ml}$ complete medium was added into the well. Following incubating for $24 \mathrm{~h}$, the media were replaced with complete medium. After another $72 \mathrm{~h}$ incubation, a stable cell line was established.

Infection with shRNA. FoxM1 shRNA was purchased from Sigma-Aldrich; Merck KGaA. Darmstadt, Germany (sequence, 5'-GGAAATGCTTGTGATTCAACA-3'). The lentivirus was used to transfect shFoxM1 into the sw480 cell line. Exponentially growing untreated cells were plated $24 \mathrm{~h}$ prior to infection. Plated cells were infected with FoxM1 shRNA or a negative control with $1 \mathrm{ml}$ of the lentivirus for $72 \mathrm{~h}$. Fluorescence microscopy and western blotting were used to ensure that the shRNA was successfully transfected into the sw480 cell line, and that FoxM1 was successfully knocked down.

Western blot analysis. Cells were seeded in $25 \mathrm{~cm}^{2}$ culture flasks $\left(2.5 \times 10^{5}\right.$ cells $/ 5 \mathrm{ml}$ medium). Following treatment, the cells were collected, washed twice in ice-cold phosphate-buffered saline and lysed in a lysis buffer at $4^{\circ} \mathrm{C}$. The total protein concentration for each sample was determined with a bicinchoninic acid kit. Western blotting was performed with $40 \mu \mathrm{g}$ for each sample by SDS-PAGE and transferred onto polyvinylidene fluoride transfer membranes for western blotting. The membranes were blocked with a blocking buffer (5\% dry milk in TBS-Tween-20) for $1 \mathrm{~h}$. After being washed with TBS-T, the membranes were probed with the following primary antibodies: FoxM1, Lc3, P62, p-H2A.X and $\beta$-actin. Following another wash with TBS-T, the membranes were incubated with anti-rabbit or anti-mouse secondary antibody. All antibodies were diluted in TBS-T containing 5\% BSA. Chemiluminescence detection was performed with X-ray film.

Cell viability and proliferation assays. Cell viability and proliferation were measured by MTT. The cells were digested with pancreatin. Following centrifugation, the cells were resuspended into fresh complete culture solution and then

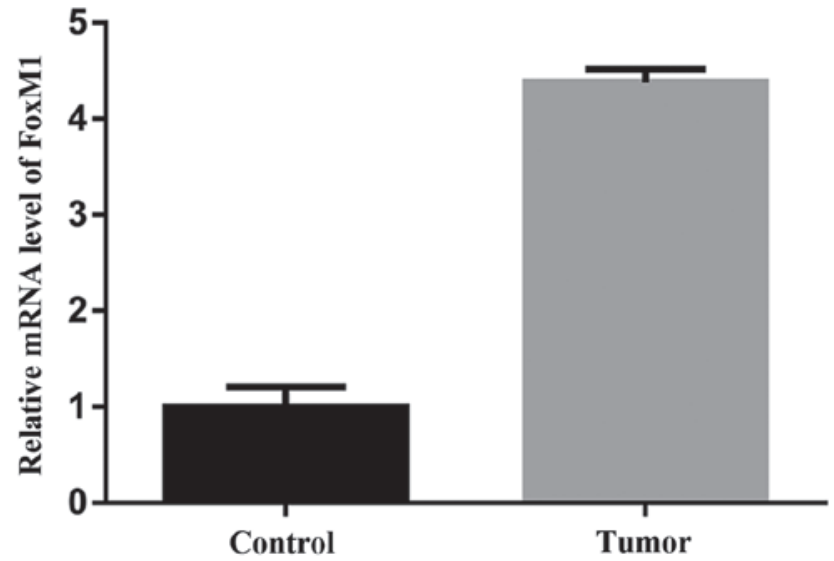

Figure 1. Relative mRNA level in tumors and paired control normal tissues. A total of five paired tumors and normal tissues were tested by reverse transcription-quantitative polymerase chain reaction. FoxM1 mRNA level in the tumor was upregulated, compared with the normal tissues. $\beta$-actin was used as a control.
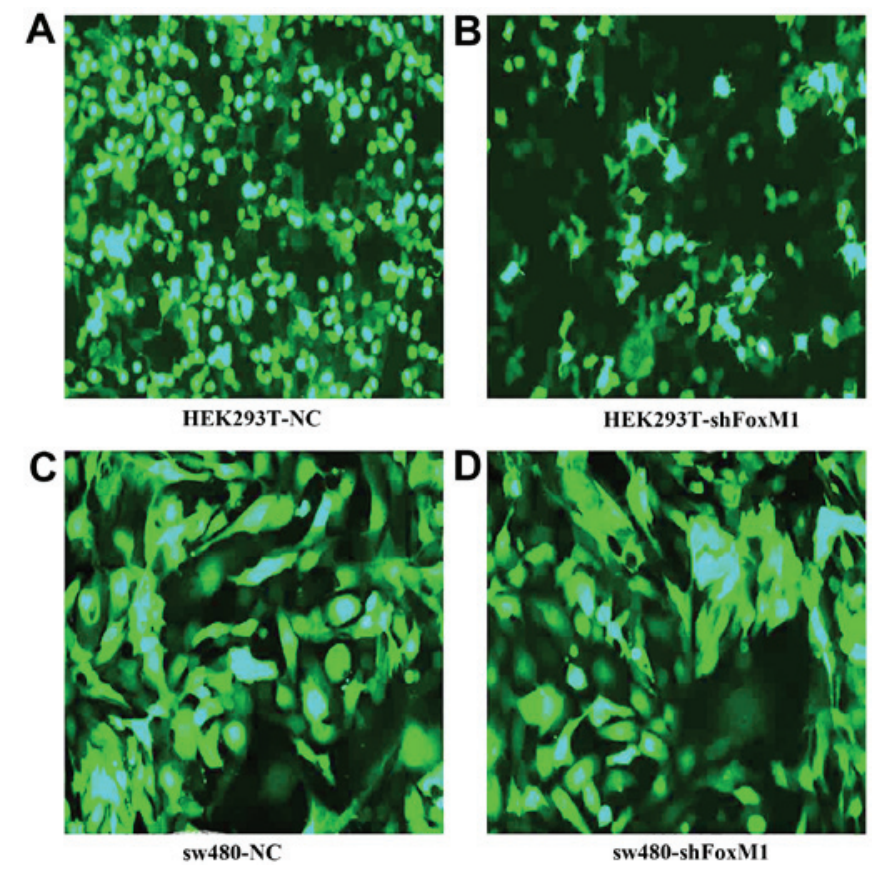

Figure 2. The lentivirus was successfully produced in HEK293T cells and transfected into the sw480 cell line. (A and B) shFoxM1 and negative control-containing lentiviruses were initially successfully transfected into HEK293T cells, and the (C and D) lentiviruses were transfected into the sw480 cell line. Fluorescence microscopy was used to detect GFP. sh, short hairpin.

seeded into a 96 -well plate $\left(5 \times 10^{3} / 200 \mu \mathrm{l}\right)$, repeating 5 -wells. Then, the cells were incubated in an incubator with $5 \% \mathrm{CO}_{2}$ at $37^{\circ} \mathrm{C}$ for $24-96 \mathrm{~h}$, and $20 \mu \mathrm{l}$ MTT solution $(5 \mathrm{mg} / \mathrm{ml})$ was added for color generation. Incubation was continued for extra $4 \mathrm{~h}$ in an incubator with $5 \% \mathrm{CO}_{2}$ at $37^{\circ} \mathrm{C}$ and the culture solution was aspirated following $4 \mathrm{~h}$. DMSO $(200 \mu \mathrm{l})$ was added into every well and the samples were slightly shaken for $10 \mathrm{~min}$ to speed up the dissolution of crystals. The absorbance value of each well was detected by MTT following 0 , $24,48,72$ and $96 \mathrm{~h}$. The absorbance value and interval time were posited as vertical coordinates and abscissa, respectively, 


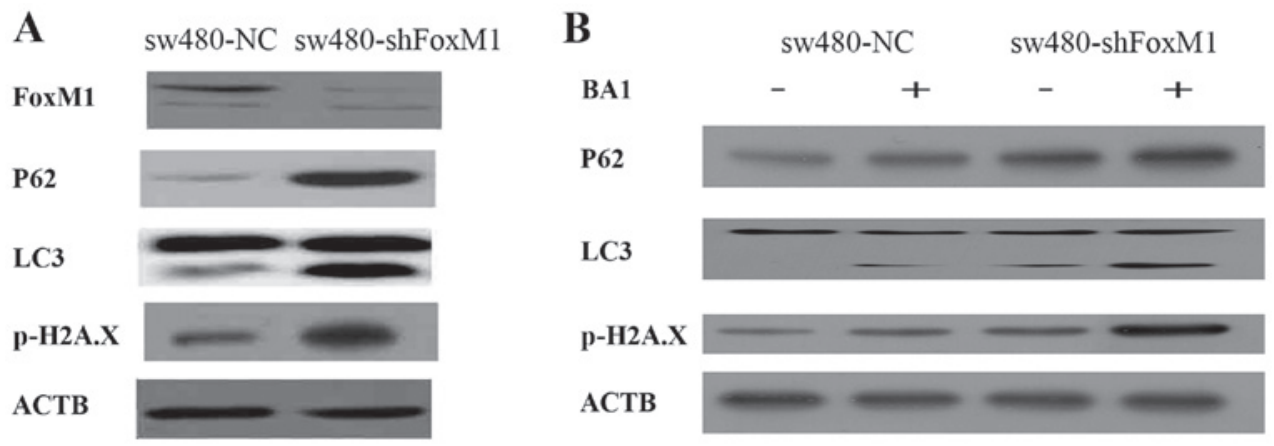

Figure 3. FoxM1 was successfully knocked down in the sw480 cell line, and downregulating FoxM1 significantly reduced autophagy level. (A) FoxM1 was successfully knocked down in the sw480 cell line. P62 and LC3 p-H2A.X were detected to measure the changes in autophagy. ACTB was used as a control (B) sw480-NC and sw480-shFoxM1 were treated with Baf A1 (10 nM) for $2 \mathrm{~h}$. Western blotting was performed to test the state of LC3 p62 H2A.X and ACTB. ACTB, $\beta$-actin; Baf 1, bafilomycin A1; NC, negative control; sh, short hairpin.

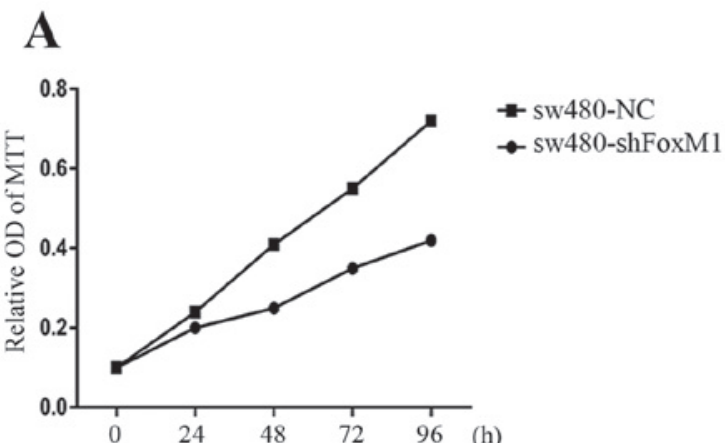

C

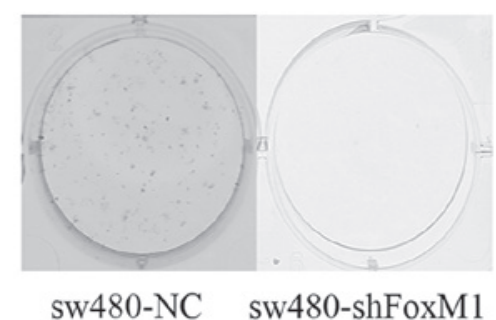

\section{B}

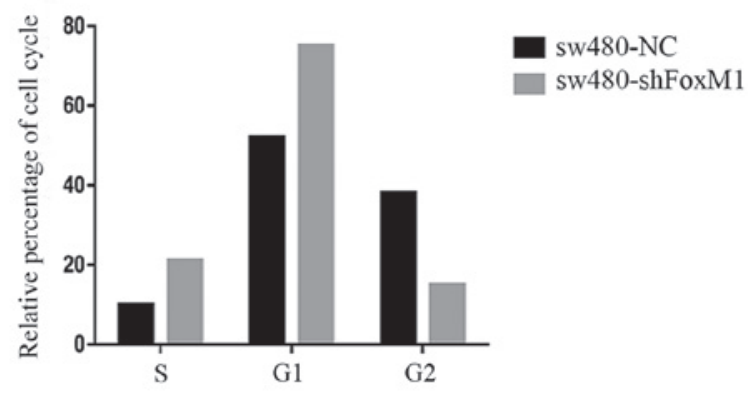

D

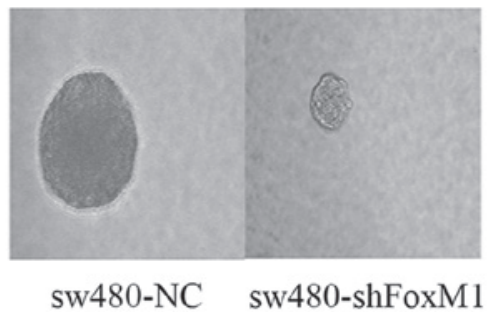

Figure 4. (A) MTT was used to examine the effort on the short-term proliferation of downregulated FoxM1. Consistently, downregulated FoxM1 reduced the proliferation in the sw480 cell line in the early stages, which is corresponded with the flow cytometry data. (B) Flow cytometry was used to test the cell cycle. Downregulating FoxM1 shows G1 arrest compared with the negative control. (C and D) Knockdown of FoxM1 inhibits cell proliferation in the sw480 cell line. A clonogenic assay was used to measure the ability to form the foci. Downregulation of FoxM1 resulted in a significantly reduction in the number of colony compared with the control group. NC, negative control; OD, optical density; sh, short hairpin.

following which, the MTT graph was drawn. All procedures were repeated three times.

Cell cycle by flow cytometry. The cycle distribution was analyzed by flow cytometry following staining with propidium iodide (PI) solution. Briefly, sw480-shFoxM1 and negative control cells were seeded into a 6 -well plate with $3 \times 10^{6} /$ well following incubating for $24 \mathrm{~h}$, cells were fixed with $75 \%$ ethanol. Next, the cells were incubated with $500 \mathrm{ml}$ solution containing $50 \mathrm{mg} / \mathrm{ml} \mathrm{PI}$ and $0.1 \%$ Triton X-100 in the dark and analyzed by flow cytometry. Finally, the data was analyzed by Flow-Job software.

Colony formation assay. Cells were trypsinized and plated in $10 \mathrm{~cm}$ dishes at a density of $5 \times 10^{1} /$ dish. Following incubating at $37^{\circ} \mathrm{C} 5 \% \mathrm{CO}_{2}$ for 2 weeks, the authors used Methylrosanilinium Chloride Solution to dye colony.

Soft-agar clonogenicity assay. Clonogenic growth of cells was evaluated by seeding $0.5 \times 10^{3}$ cells in $0.5 \mathrm{ml}$ RPMI-1640 supplemented with $0.33 \%$ agar and $10 \%$ FBS. Cells were grown in vitro $\left(37^{\circ} \mathrm{C}, 5 \% \mathrm{CO}_{2}\right)$ for 3 weeks.

\section{Results}

FoxM1 is significantly upregulated in colon carcinoma compared with paired normal tissues. FoxM1 was upregulated in colon cancers, when compared with normal tissues in many published researches at both mRNA and protein level and attributes to the tumorigenesis of colon cancer (Fig. 1). 
shFoxM1 and the negative control lentivirus was successfully produced in HEK293T cells and was successfully infected into the sw 480 cell line. To investigate the potential function of FoxM1 in the sw480 cell line, the expression level of FoxM1 was knocked down by using a lentivirus system. Following infection for 3 days, after screening with puromycin for 3 days. Fluorescence microscopy was used to detect GFP and the infection efficiency. $99 \%$ cells were successfully infected with lentivirus (Fig. 2).

FoxM1 was successfully knocked down in the sw 480 cell line and downregulating FoxM1 significantly reduced autophagy level. Following establishing the stable shFoxM1 cell line, the authors investigated the relationship between downregulating FoxM1 and autophagy level.LC3 p62 and p-H2A.X were tested (Fig. 3A). p62 is a selective substrate for autophagy-lysosome degradation, so, total p62 protein levels reflect autophagic activity. To test whether the decrease in autophagosomes is due to decreased autophagy activity or a block in downstream degradation, The authors performed an autophagic flux assay. Bafilomycin A1 (Baf A1) is a lysosomotropic reagent that blocks autophagosome degradation. As was expected, Baf A1 treatment caused elevated levels of LC 3 in the NC, shFoxM1 cells. In addition, p62 protein levels increased upon Baf A1 treatment in the NC, shFoxM1 cells (Fig. 3B). Therefore, it may be concluded that downregulating FoxM1 decreases autophagic activity.

Knockdown of FoxM1 inhibits cell proliferation in the sw 480 cell line. Following successfully FoxM1, the authors conducted a series of experiments to test FoxM1-mediated biological function. A clonogenic assay was used to measure the ability to form the foci. Downregulation of FoxM1 resulted in a significantly reduction in the number of colonies compared with the control group (Fig. 4C and D). Flow cytometry was used to test the cell cycle. Downregulation of FoxM1 indicated G1 arrest compared with the negative control (Fig. 4B). MTT were used to examine the effort on the short-term proliferation of downregulated FoxM1. Consistently, downregulated FoxM1 reduced the proliferation in the sw480 cell line in the early stage, which is corresponded with the flow cytometry results (Fig. 4A).

\section{Discussion}

Colon cancer is one of the most frequently diagnosed cancers and has been well studied in the past decades. FoxM1, a member of the Fox protein family, characterized by a conserved winged-helix DNA binding domain, promotes cell cycle progression by inducing both transition from $\mathrm{G} 1$ to $\mathrm{S}$ phase and transition from G2 to M phase. Autophagy is one of the most prominently studied fields worldwide, however, the mechanism remains unclear in the sw480 cell line; the authors aimed to uncover its mechanism in the present study.

A previous study of the authors demonstrated the correlation between FoxM1 expression and autophagy level though detecting LC3B and P62. The ratio of LC3BII to LC3BI increased and P62 significantly accumulated in sw480-shFoxM1, indicating that the autophagy level was declined. Furthermore, Baf A1 is a lysosomotropic reagent that blocks autophagosome degradation. As expected, Baf A1 treatment caused elevated levels of LC3 in the NC, shFoxM1 cells. In addition, p62 protein levels increased upon Baf A1 treatment in the NC, shFoxM1 cells. Therefore, downregulating FoxM1 decreases autophagic activity.

The damage of DNA was enhanced, the accumulated p-H2A.X also confirmed this conclusion. In the current research, it was hypothesized that knockdown of FoxM1 may exert its function by upregulating DNA damage and downregulating autophagy level through inhibiting the recycling of many organelles to affect tumor tumorigenesis.

In conclusion, the present results demonstrated the relationship between downregulating FoxM1 and autophagy and its potential mechanism. Downregulating FoxM1 significantly reduced autophagy level and enhanced DNA damage to inhibit proliferation in the sw480 cell line.

\section{References}

1. Fang YJ, Wu XJ, Zhao Q, Li LR, Lu ZH, Ding PR, Zhang RX, Kong LH, Wang FL, Lin JZ, et al: Hospital-based colorectal cancer survival trend of different tumor locations from 1960s to 2000s. PLoS One 8: e73528, 2013.

2. Prasetyanti PR, Capone E, Barcaroli D, D'Agostino D, Volpe S, Benfante A, van Hooff S, Iacobelli V, Rossi C, Iacobelli S, et al: ErbB-3 activation by NRG-1 $\beta$ sustains growth and promotes vemurafenib resistance in BRAF-V600E colon cancer stem cells (CSCs). Oncotarget 6: 16902-16911, 2015.

3. Anders L, Ke N, Hydbring P, Choi YJ, Widlund HR, Chick JM, Zhai H, Vidal M, Gygi SP, Braun P, et al: A systematic screen for CDK4/6 substrates links FOXM1 phosphorylation to senescence suppression in cancer cells. Cancer Cell 20: 620-634, 2011.

4. Bao B, Wang Z, Ali S, Kong D, Banerjee S, Ahmad A, Li Y, Azmi AS, Miele L and Sarkar FH: Over-expression of FoxM1 leads to epithelial-mesenchymal transition and cancer stem cell phenotype in pancreatic cancer cells. J Cell Biochem 112: 2296-2306, 2011.

5. Kalin TV, Wang IC, Ackerson TJ, Major ML, Detrisac CJ, Kalinichenko VV, Lyubimov A and Costa RH: Increased levels of the FoxM1 transcription factor accelerate development and progression of prostate carcinomas in both TRAMP and LADY transgenic mice. Cancer Res 66: 1712-1720, 2006.

6. Wierstra I and Alves J: FOXM1, a typical proliferation-associated transcription factor. Biol Chem 388: 1257-1274, 2007.

7. Yu J, Deshmukh H, Payton JE, Dunham C, Scheithauer BW, Tihan T, Prayson RA, Guha A, Bridge JA, Ferner RE, et al: Array-based comparative genomic hybridization identifies CDK4 and FOXM1 alterations as independent predictors of survival in malignant peripheral nerve sheath tumor. Clin Cancer Res 17: 1924-1934, 2011.

8. Barsotti AM and Prives C: Pro-proliferative FoxM1 is a target of p53-mediated repression. Oncogene 28: 4295-4305, 2009.

9. Levine B and Kroemer G: Autophagy in the pathogenesis of disease. Cell 132: 27-42, 2008.

10. Lum JJ, Bauer DE, Kong M, Harris MH, Li C, Lindsten T and Thompson CB: Growth factor regulation of autophagy and cell survival in the absence of apoptosis. Cell 120: 237-248, 2005.

11. Pattingre S, Tassa A, Qu X, Garuti R, Liang XH, Mizushima N, Packer M, Schneider MD and Levine B: Bcl-2 antiapoptotic proteins inhibit Beclin 1-dependent autophagy. Cell 122: 927-939, 2005.

12. Mathew R, Karp CM, Beaudoin B, Vuong N, Chen G, Chen HY, Bray K, Reddy A, Bhanot G, Gelinas C, et al: utophagy suppresses tumorigenesis through elimination of p62. Cell 137: 1062-1075, 2009. 